\title{
Finite element method for optimum design selection of carport structures under multiple load cases
}

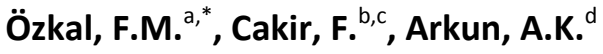 \\ ${ }^{a}$ Erzincan University, Department of Civil Engineering, Erzincan, Turkey \\ ${ }^{b}$ Yıldız Technical University, Department of Architecture, İstanbul, Turkey \\ 'University of California, Pacific Earthquake Engineering Research Center (PEER), Berkeley, California, USA \\ ${ }^{\mathrm{d}}$ Amasya University, Department of Urban Design and Landscape Architecture, Amasya, Turkey
}

\section{A B S T R A C T}

In the field of structural modelling, it is obvious that the number of applicable designs for a particular structural necessity is limitless. Along with the integration of various kinds of available structural materials into this complexity, it gets harder to be able to determine the best design before the production stage. In recent years, with the improvement of computational and structural technology, there have been many studies on the optimal design selection. This study focuses on carport structures and pursuing their best producible shape. For this aim, a performance index formulation was developed to assist the decision of material efficiency as well as structural rigidity. Thereafter, five conceptual models were numerically modelled and finite element analyses (FEA) for multiple load cases were carried out. Reviewing the FEA results, the most appropriate model was determined by the application of this performance qualification method. Results of the analyses show that optimum design of structures under multiple load cases can be determined using finite element method.
\end{abstract}

(c) 2016 PEI, University of Maribor. All rights reserved.

\section{ARTICLE INFO}

\section{Keywords:}

Structural producibility

Performance decision

Multiple load cases

Manufacturing

Finite element method

*Corresponding author: fmozkal@erzincan.edu.tr (Özkal, F.M.)

Article history:

Received 26 April 2016

Revised 5 October 2016

Accepted 12 October 2016

\section{References}

[1] Kosheleva, O., Kreinovich, V., Nguyen, H.T. (2015). Why it is important to precisiate goals. Departmental Technical Reports (CS), Technical Report: UTEP-CS-15-25, Paper 920.

[2] Kirsch, U. (1981). Optimum structural design, McGraw Hill, New York, USA.

[3] Cash, P., Hicks, B., Culley, S. (2015). Activity Theory as a means for multi-scale analysis of the engineering design process: A protocol study of design in practice. Design Studies, Vol. 38, 1-32, doi: 10.1016/i.destud.2015.02.001.

[4] Haldankar, M., Shirahatti, A.M. (2014). Finite element analysis and optimization of commercial bus body structure, International Journal of Engineering and Technical Research, Vol. 2, No. 12, 175-178.

[5] Jain, R., Tandon, P., Kumar, M.V. (2014). Optimization methodology for beam gauges of the bus body for weight reduction, Applied and Computational Mechanics, Vol. 8, No. 1, 47-62.

[6] Srihari, P., Azad, D., Sreeramulu, D. (2014). Optimization of rail inserts using finite element analysis. International Journal of Engineering, Science and Technology, Vol. 6, No. 2, 65-75, doi: 10.4314/ijest.v6i2.5.

[7] Marczak, R.J. (2007). Optimization of elastic structures using boundary elements and a topological-shape sensitivity formulation, Latin American Journal of Solids and Structures, Vol. 5, No. 2, 99-117.

[8] Rajan, S.D., Belegundu, A.D., Lee, D., Damle, A.S., St Ville, J. (2004). Finite element analysis \& design optimization in a distributed computing environment, In: Collection of Technical Papers - $10^{\text {th }}$ AIAA/ISSMO Multidisciplinary Analysis and Optimization Conference, Albany, NY, USA, Vol. 3, 1716-1726.

[9] Moore, A. Carport History, from https://www.versatube.com/news/carport-history accessed January 30, 2016.

[10] Gebhard, D. (1992). The suburban house and the automobile, In: Wachs, M., Crawford, M. (ed.), The car and the city: the automobile, the built environment and daily urban life, Ann Arbor, University of Michigan Press, Michigan, USA, 106-123. 
[11] Fox, J., Jeffery, R.B. (2005). Carport Integrity Policy, Arizona State Historic Preservation Office, Preservation Studies, University of Arizona, USA, 1-9.

[12] Robinson, M. (2000). Taj Mahal on a Cul de Sac: Concrete blocks, carports and architectural appropriation, Arris - The Journal of the Southeast Chapter of the Society of Architectural Historians, Vol. 11, 71-83.

[13] Anonymous. Vintage Designs, Gaineswood, from http://vintagedesigns.com/architecture/gkrev/gwd/index.htm. accessed January 29, 2016.

[14] Anonymous. Auto Space, The Garage Journal, from http://www.garagejournal.com/2009/03/auto-space, accessed January 29, 2016.

[15] Anonymous. Affordable, Modern, Sustainable Homes, http://www.archdaily.com/238195/connecthomes-offersaffordable-modern-sustainable-homes/4series carport, accessed January 29, 2016.

[16] Anonymous. Carport Collapse (Spokane), from http://images.fanpop.com/images/image uploads/Winter-in-theInland-Northwest-winter-708370 450 299.jpg accessed January 21, 2016.

[17] Anonymous. Imgarcade, Homemade Carport Collapse, from http://imgarcade.com/1/homemade-carport, accessed January 21, 2016.

[18] Stickney, R., NBC San Diego, Collapsed Carport Crushes Cars in Alpine, from http://www.nbcsandiego.com/news/local/Carport-Collapse-Alpine-Arnold-Way-San-Diego-Heartland-234238281.html, accessed January 21, 2016.

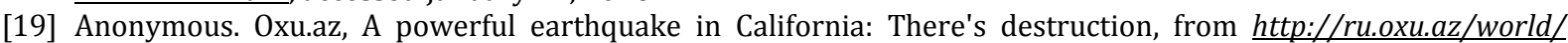
39156, accessed January 21, 2016.

[20] ANSYS (2014). Releases 14.0, Finite element analysis software, USA.

[21] Özkal, F.M., Uysal, H. (2012). A fully stressed design method to determine the optimum strut-and-tie model for beam-column connections. International Journal of Computational Methods, Vol. 9, No. 3, 1250035, doi: $10.1142 / \mathrm{S} 0219876212500351$.

[22] Manifold, S.M. (2014). Stability analysis and finite element stress analysis of a solarwing carport structure and solar panel array, Technical Report: Concurrent Design, Inc., from http://www.concurrentdesign.com/engineeringanalysis.htm, accessed September 28, 2016.

[23] Hrestak, T., Lazarević, A.J., Frgić, L. (2015). Stress and strain analysis during the Sleme tunnel excavation, Technical Gazette - Tehnički Vjesnik, Vol. 22, No. 3, 703-709, doi: 10.17559/TV-20140530103847. 


\section{APEM}

\title{
Metoda končnih elementov za optimalno zasnovo konstrukcije avtomobilskih nadstreškov pri različnih obremenitvah
}

\author{
Özkal, F.M. ${ }^{a,}{ }^{*}$, Cakir, F. ${ }^{b, c}$, Arkun, A.K. ${ }^{d}$ \\ ${ }^{a}$ Erzincan University, Department of Civil Engineering, Erzincan, Turkey \\ ${ }^{b}$ Yıldız Technical University, Department of Architecture, İstanbul, Turkey \\ 'University of California, Pacific Earthquake Engineering Research Center (PEER), Berkeley, California, USA \\ ${ }^{d}$ Amasya University, Department of Urban Design and Landscape Architecture, Amasya, Turkey
}

\begin{abstract}
POVZETEK
Pri zasnovi konstrukcij je strukturnih rešitev ne glede na potrebe praktično neomejeno. Ko v zasnovo konstrukcije vključimo različne razpoložljive materiale, pa je najboljšo strukturo še težje določiti. V preteklem obdobju je bilo na to temo opravljenih dosti študij. Ta prispevek se ukvarja s določitvijo oblike konstrukcije za avtomobilske nadstreške z ozirom na prednosti pri proizvodnji. Vpeljan je indeks uspešnosti, ki pomaga pri izbiri materiala in strukturne togosti. Pripravljenih je bilo pet numeričnih modelov za preračun z metodo končnih elementov (MKE) pri različnih obremenitvah. Iz rezultatov MKE in z uporabo uspešnostne kvalifikacijske metode je nato izbran najprimernejši koncept. Rezultati kažejo, da je s pomočjo MKE mogoče določiti najboljšo strukturno zasnovo konstrukcije pri različnih obremenitvah.
\end{abstract}

(C) 2016 PEI, University of Maribor. All rights reserved.

\section{PODATKI O ČLANKU}

Ključne besede: Izvedljivost strukture

Izbira po uspešnosti

Obremenitveni testi

Proizvodnja

Metoda končnih elementov

*Kontaktna oseba:

fmozkal@erzincan.edu.tr

(Özkal, F.M.)

Zgodovina članka:

Prejet 26. aprila 2016

Popravljen 5. oktobra 2016

Sprejet 12. oktobra 2016 